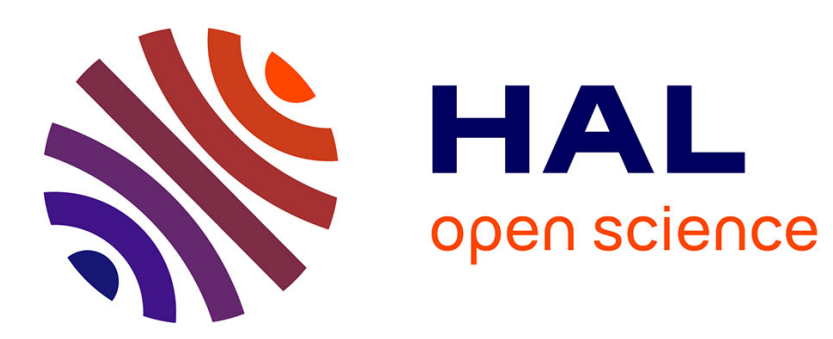

\title{
Generic uniform observability analysis for bilinear systems
}

Taha Boukhobza

\section{To cite this version:}

Taha Boukhobza. Generic uniform observability analysis for bilinear systems. Automatica, 2008, 44 (12), pp.3133-3138. 10.1016/j.automatica.2008.05.012 . hal-00338115

\section{HAL Id: hal-00338115 https://hal.science/hal-00338115}

Submitted on 11 Nov 2008

HAL is a multi-disciplinary open access archive for the deposit and dissemination of scientific research documents, whether they are published or not. The documents may come from teaching and research institutions in France or abroad, or from public or private research centers.
L'archive ouverte pluridisciplinaire HAL, est destinée au dépôt et à la diffusion de documents scientifiques de niveau recherche, publiés ou non, émanant des établissements d'enseignement et de recherche français ou étrangers, des laboratoires publics ou privés. 


\title{
Generic uniform observability analysis for bilinear systems
}

\author{
T. Boukhobza \\ Centre de Recherche en Automatique de Nancy (CRAN - CNRS UMR 7039), Nancy University, \\ BP 239, 54506 Vandouvre Cedex, Nancy, France, \\ email: taha.boukhobza@cran.uhp-nancy.fr
}

\begin{abstract}
In this paper, we study the property of generic uniform observability for structured bilinear systems. More precisely, to check whether or not a structured bilinear system generically uniformly observable, we provide a group of necessary conditions and second group of sufficient ones. These conditions are expressed in quite simple graphic-terms. On the one hand, all the given conditions are easy to check because they deal with finding paths in a digraph. On the other hand, the proposed method is based on a graph-theoretic approach and assumes only the knowledge of the system's structure. This makes the suggested approach particularly well suited to study large scale systems or systems with unknown parameters, as it may be the case during a conception stage.
\end{abstract}

Key words: Structured bilinear systems, generic uniform observability, graph theory.

\section{Introduction}

The class of bilinear systems (BLS), representing particular nonlinear systems whose dynamics are jointly linear in the state and the input variables, was introduced in control theory in the 1960's. Many works deal with bilinear systems, because this class of systems are simpler and better understood than most other nonlinear systems. Furthermore, industrial process control, economics and biology (switched circuits, mechanical brakes, controlled suspension systems, immunological systems, population growth, enzyme kinetics, ... ) provide lot of examples of BLS (Mohler, 1991).

The observability of these systems is tackled in many works among which we can cite (Williamson, 1977; Grasselli and Isidori, 1977). Theoretically, the observability notion is based on the concept of distinguishability of two initial states using the knowledge of the measurements and input values. However, for nonlinear systems, the distinguishability of two initial states depends on the input applied to the system. In this respect, a BLS is observable if it can be found an input such that any pair of initial states are distinguishable by observation of the corresponding outputs (Williamson, 1977; Kou et al., 1996). From the latter definition, the notion of universal input is defined in (Gauthier and Bornard, 1981; Hermann and Krener, 1977) as an input for which every pair of initial states can be distinguished by observation of the corresponding outputs. A system, for which every admissible input is universal is said uniformly observable. As it is the case for the notion of strong observability (Trentelman et al., 2001) for linear systems, the analysis of the uniform observability is important since it informs us on the possible alteration of the state distinguishability for some input values. This information is useful obviously for observer design and also for control, fault detection and isolation schemes which all use the estimated state variables. Many studies are dedicated to the analysis of the uniform observability (Williamson, 1977; Gauthier and Bornard, 1981; Gauthier and Kupka, 1994). In most cases, either the authors are interested only in the single output case or they propose sufficient conditions to achieve uniform observability. In the single output case, a triangular canonical form for uniformly observable systems is provided in (Gauthier and Bornard, 1981). Otherwise, the necessary and sufficient conditions given in (Williamson, 1977) are quite trivial and mainly difficult to apply to large scale systems because they are based on the computation of the rank of the observability matrix in function of the input values.

The latter results, as many others concerning bilinear systems, are based on geometric or algebraic approaches. However, the use of such tools assumes the exact knowledge of the state space matrices characterizing the system's model. In many modeling problems, these matrices have a number of fixed zero entries determined by the physical laws while the remaining entries are not precisely known. To analyse the properties of these systems in spite of poor knowledge we have on them, the idea is that we only keep the zero/non-zero entries in the state space matrices. Thus, we consider models, called structured models, where the fixed zeros are conserved while the non-zero entries are replaced by free parameters. The studies on structured systems are often related to the graph-theoretic approach which allows to obtain analysis tools for some properties such as controllability, observability and the solvability of several classical control problems including disturbance rejection, input-output decoupling, ....(see (Dion et al., 2003) and the references therein). It results from these works that the graph-theoretic approach provides simple and elegant solutions requiring a low computational burden and so are well adapted for large scale systems.

Unfortunately, few works based on graph-theoretic methods deal with nonlinear systems. Among them, (Svaricek, 1993) gives sufficient conditions to fulfill the observability of 
bilinear systems, (Lévine, 1997) study the input-output decoupling and linearization of a nonlinear system and more recently, (Bornard and Hammouri, 2002) proposes graphical sufficient conditions for checking the uniform observability of nonlinear systems which are preliminarily put in a canonical form. Finally, in (Boukhobza et al., 2006) we give a first analysis tool of the uniform observability condition. In this context, the purpose of this paper is to improve the results presented in (Boukhobza et al., 2006), which are quite complicated from a computational point of view and, for some cases, do not allow to conclude whether or not a system is generically uniformly observable. In the present paper, we present two groups of graphical conditions (the first ones are necessary while the others are sufficient) to check the uniform observability of structured bilinear systems. These conditions need few information about the system and are easy to check by means of wellknown combinatorial techniques. Our results are applicable to multi-output systems and the proposed conditions become necessary and sufficient in the case of single-output systems. They are adapted for large-scale systems and there is no need to put the system in any canonical form before applying the proposed method.

The paper is organised as follows: after Section 2, which is devoted to the problem formulation, a digraph representation of structured bilinear system (SBLS) is given in Section 3. The main result of the paper is provided in Section 4. Finally, some concluding remarks are made.

\section{Problem statement}

In this paper, we consider SBLS in the form:

$$
\Sigma_{\Lambda}:\left\{\begin{array}{l}
\dot{x}=A x+\sum_{i=1}^{m} u_{i} B_{i} x \\
y=C x
\end{array}\right.
$$

where $x=\left(x_{1}, \ldots, x_{n}\right)^{T} \in \mathbb{R}^{n}, u=\left(u_{1}, \ldots, u_{m}\right)^{T} \in \mathbb{R}^{m}$ and $y=\left(y_{1}, \ldots, y_{p}\right)^{T} \in \mathbb{R}^{p}$ are respectively the state, the input and the output vectors. $A, C$ and $B_{i}$ for $i=1, \ldots, m$ matrices of appropriate dimensions. We assume that only the zero/nonzero structure of $A, B_{i}$, for $i=1, \ldots, m$, and $C$ is known. This means that, to each entry in these matrices, we only know whether its value is fixed to zero, in which case we call it a fixed zero, or that it has an unknown real value, in which case we call this entry a free parameter. In a structured system with $h$ nonzero entries in $A, B_{i}$, for $i=1, \ldots, m$, and $C$, we can parameterize these nonzero entries by scalar real (nonzero) parameters $\lambda_{j}, j=1, \ldots, h$ forming a parameter vector $\Lambda=\left(\lambda_{1}, \ldots, \lambda_{h}\right)^{T} \in \mathbb{R}^{h}$. If all parameters $\lambda_{j}$ are numerically fixed, we obtain a so-called admissible realization of structured system $\Sigma_{\Lambda}$.

We say that a property is true generically (van der Woude, 2000) if it is true for almost all the realizations of structured system $\Sigma_{\Lambda}$. Here, "for almost all the realizations" is to be understood as "for all parameter values $\left(\Lambda \in \mathbb{R}^{h}\right)$ except for those in some proper algebraic variety in the parameter space". The proper algebraic variety for which the property is not true is the zero set of some nontrivial polynomial with real coefficients in the system parameters.

In order to precise the notion of generic uniform observability, we adapt the definition of the uniform observability (Williamson, 1977; Gauthier and Bornard, 1981) to the SBLS:

Definition 1 Structured bilinear system $\Sigma_{\Lambda}$ is generically uniformly observable if, for almost all its realizations and for any admissible input, any pair of initial states $x^{0}(0)$ and $x^{1}(0)$ are distinguishable by observation of the corresponding outputs $y^{0}(t)$ and $y^{1}(t)$ for $t \geq 0$.

In the present work, using a graph-theoretic approach, we propose an analysis tool for studying the generic observability of SBLS $\Sigma_{\Lambda}$.

\section{Graphical representation of a SBLS}

This section is devoted to the definition of the digraph which is used to represent SBLS $\Sigma_{\Lambda}$. This digraph is noted $G\left(\Sigma_{\Lambda}\right)$ and is constituted by a vertex set $\mathcal{V}$ and an edge set $\mathcal{E}$. More precisely, the vertex set is defined by: $\mathcal{V}=\mathbf{X} \cup \mathbf{U}_{\mathbf{e}} \cup \mathbf{U} \cup \mathbf{Y}$, where $\mathbf{X}=\left\{\mathbf{x}_{1}, \ldots, \mathbf{x}_{\mathbf{n}}\right\}$ is the set of state vertices, $\mathbf{U}=\left\{\mathbf{u}_{1}, \ldots, \mathbf{u}_{\mathbf{m}}\right\}$ is the set of input vertices, $\mathbf{U}_{\mathbf{e}}=\left\{\mathbf{u}_{\mathbf{i}, \mathbf{j}}\right.$, if $\left.\exists \ell=1, \ldots, n \mid B_{i}(\ell, j) \neq 0\right\}$ is the set of extended input vertices representing the products $u_{i} x_{j}$ present in the model of the system and $\mathbf{Y}=\left\{\mathbf{y}_{\mathbf{1}}, \ldots, \mathbf{y}_{\mathbf{p}}\right\}$ is the set of output vertices. The edge set is $\mathcal{E}=\bigcup_{i=0}^{m} A_{i}$-edges $\cup C$-edges $\cup$ $B$-edges $\cup B_{u}$-edges with $C$-edges $=\left\{\left(\mathbf{x}_{\mathbf{j}}, \mathbf{y}_{\mathbf{i}}\right) \mid C(i, j) \neq 0\right\}$, $A_{0}$-edges $=\left\{\left(\mathbf{x}_{\mathbf{k}}, \mathbf{x}_{\mathbf{j}}\right) \mid A(j, k) \neq 0\right\}$, for $i=1, \ldots, m, A_{i}$-edges $=$ $\left\{\left(\mathbf{x}_{\mathbf{k}}, \mathbf{x}_{\mathbf{j}}\right) \mid B_{i}(j, k) \neq 0\right\}, \quad B$-edges $=\left\{\left(\mathbf{u}_{\mathbf{i}, \mathbf{j}}, \mathbf{x}_{\mathbf{k}}\right) \mid B_{i}(k, j) \neq 0\right\}$ and $B_{u}$-edges $=\left\{\left(\mathbf{u}_{\mathbf{i}}, \mathbf{u}_{\mathbf{i}, \mathbf{j}}\right), \forall i=1, \ldots, m\right.$ and $\forall j=1, \ldots n$ if $\left.\exists \ell \mid B_{i}(\ell, j) \neq 0\right\}$.

Here $M(i, j)$ is the $(i, j)$ th element of matrix $M$ and $\left(\mathbf{v}_{\mathbf{1}}, \mathbf{v}_{\mathbf{2}}\right)$ denotes a directed edge from vertex $\mathbf{v}_{\mathbf{1}} \in \mathcal{V}$ to vertex $\mathbf{v}_{\mathbf{2}} \in \mathcal{V}$. Note that, we indicate the number $i$ under each $A_{i}$-edge in order to preserve the information about the belonging of the edges in the digraph representation.

Hereafter, we illustrate our proposed digraph representation for SBLS with a simple example:

Example 2 Consider the SBLS defined by:

$$
\begin{aligned}
A & =\left(\begin{array}{ccccc}
0 & \lambda_{1} & \lambda_{2} & 0 & 0 \\
0 & 0 & 0 & \lambda_{3} & 0 \\
0 & 0 & 0 & 0 & \lambda_{4} \\
0 & 0 & 0 & 0 & 0 \\
0 & 0 & 0 & 0 & \lambda_{5}
\end{array}\right), B_{1}=\left(\begin{array}{ccccc}
0 & 0 & 0 & 0 & 0 \\
0 & 0 & 0 & 0 & \lambda_{6} \\
0 & 0 & \lambda_{7} & 0 & 0 \\
0 & 0 & 0 & 0 & 0 \\
0 & 0 & 0 & 0 & 0
\end{array}\right), B_{2}=\left(\begin{array}{lllll}
0 & \lambda_{8} & 0 & 0 & 0 \\
0 & 0 & 0 & 0 & 0 \\
0 & 0 & 0 & 0 & 0 \\
0 & 0 & 0 & 0 & 0 \\
0 & 0 & 0 & 0 & 0
\end{array}\right) \text { and } \\
C & =\left(\begin{array}{ccccc}
\lambda_{9} & 0 & 0 & 0 & 0 \\
0 & \lambda_{10} & 0 & 0 & 0
\end{array}\right) \text { or by the following state-space model }
\end{aligned}
$$

$$
\begin{cases}\dot{x}_{1}=\lambda_{1} x_{2}+\lambda_{2} x_{3}+\lambda_{8} u_{2} x_{2} ; & y_{1}=\lambda_{9} x_{1} \\ \dot{x}_{2}=\lambda_{3} x_{4}+\lambda_{6} u_{1} x_{5} ; & y_{2}=\lambda_{10} x_{2} \\ \dot{x}_{3}=\lambda_{4} x_{5}+\lambda_{7} u_{1} x_{3} & \\ \dot{x}_{4}=0 & \\ \dot{x}_{5}=\lambda_{5} x_{5} & \end{cases}
$$


This system is associated to the graph depicted in Figure 1.

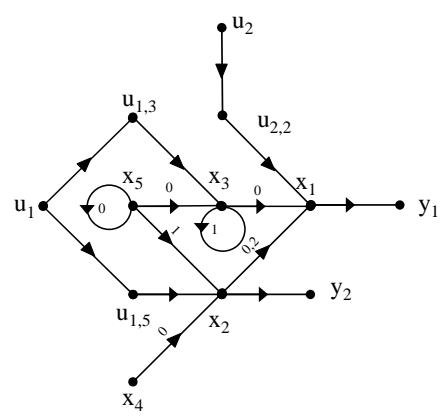

Figure 1. Digraph associated to Example 2

There are five vertices related to the state components $x_{1}, x_{2}$, $x_{3}, x_{4}$ and $x_{5}$, two vertices related to the output components $y_{1}$ and $y_{2}$, two vertices corresponding to the input components $u_{1}$ and $u_{2}$. The extended input vertices $u_{1,3}, u_{1,5}$ and $u_{2,2}$ representing respectively the products $u_{1} x_{3}, u_{1} x_{5}$ and $u_{2} x_{2}$ present in the state space model. Each vertex $\mathbf{u}_{\mathbf{i}}$ is connected to each of the extended input $\mathbf{u}_{\mathbf{i}, \mathrm{j}}$ when the latter exists. Thus, for the considered system $\mathbf{u}_{\mathbf{1}}$ is linked to $\mathbf{u}_{\mathbf{1}, \mathbf{3}}$ and $\mathbf{u}_{1,5}$ and $\mathbf{u}_{2}$ is linked to $\mathbf{u}_{2,2}$. These relations reflect how input $u_{i}$ intervene on the system dynamics. Obviously, extended vertices $u_{i, j}$ cannot exist in the usual graphical representations of linear systems. Moreover, each dynamic equation is represented by edges between the concerned variables. For example, the dynamic equation $\dot{x}_{2}=\lambda_{1} x_{2}+\lambda_{2} x_{3}+\lambda_{8} u_{2} x_{2}$ is represented by edges $\left(\mathbf{x}_{\mathbf{2}}, \mathbf{x}_{\mathbf{1}}\right),\left(\mathbf{x}_{\mathbf{3}}, \mathbf{x}_{1}\right)$ and $\left(\mathbf{u}_{2,2}, \mathbf{x}_{1}\right)$. Note that, in order to conserve in the graph the maximal information concerning the model, the edges between the state components have been indexed. Edge $\left(\mathbf{x}_{3}, \mathbf{x}_{1}\right)$ representing the term $\lambda_{2} x_{3}$ which does not depend on the inputs has the index 0 while edge $\left(\mathbf{x}_{2}, \mathbf{x}_{1}\right)$ representing the term $\lambda_{1} x_{2}+\lambda_{8} u_{2} x_{2}$ has the index 0,2 because the presence of a term independent of the input and a term depending on $u_{2}$. Finally, the output relations are represented by edges between the state vertices and the output ones. For the present system, edges $\left(\mathbf{x}_{1}, \mathbf{y}_{1}\right)$ and $\left(\mathbf{x}_{2}, \mathbf{y}_{2}\right)$ are related respectively to the relations $y_{1}=\lambda_{9} x_{1}$ and $y_{2}=\lambda_{10} x_{2}$.

Let us give some useful notations and definitions for the sequel of the paper.

- We denote by $\mathcal{E}_{0}$ the particular edge subset constituted by $\mathcal{E}$ from which we remove all $A_{i}$-edges, $i=1, \ldots, m$ i.e. $\mathcal{E}_{0}=A_{0}$-edges $\cup C$-edges $\cup B_{u}$-edges $\cup B$-edges.

- We denote path $P$ which covers $\mathbf{v}_{\mathbf{r}_{0}}, \mathbf{v}_{\mathbf{r}_{1}}, \ldots, \mathbf{v}_{\mathbf{r}_{\mathbf{i}}}$ by $P=\mathbf{v}_{\mathbf{r}_{\mathbf{0}}} \rightarrow \mathbf{v}_{\mathbf{r}_{1}} \rightarrow \ldots \rightarrow \mathbf{v}_{\mathbf{r}_{\mathbf{i}}}$, where $\left(\mathbf{v}_{\mathbf{r}_{\mathbf{j}}}, \mathbf{v}_{\mathbf{r}_{\mathbf{j}+1}}\right) \in \mathcal{E}$ for $j=0,1 \ldots, i-1$. A path is simple when every vertex in the path occurs only once. A cycle is a path of the form $\mathbf{v}_{\mathbf{r}_{0}} \rightarrow \mathbf{v}_{\mathbf{r}_{1}} \rightarrow \ldots \rightarrow \mathbf{v}_{\mathbf{r}_{\mathbf{i}}} \rightarrow \mathbf{v}_{\mathbf{r}_{0}}$, where all vertices $\mathbf{v}_{\mathbf{r}_{0}}, \mathbf{v}_{\mathbf{r}_{1}}, \ldots$, $\mathbf{v}_{\mathbf{r}_{\mathbf{i}}}$ are distinct. A path $P$ is said an $\mathbf{Y}$-topped path if its end vertex belongs to vertex subset $\mathbf{Y}$.

- Some paths are disjoint if they have no common vertex.

- An Y-topped path family consists of mutually disjoint Y-topped paths. A set of disjoint cycles is called a cycle family. The union of a $\mathbf{Y}$-topped path family and a cycle family is disjoint if the latter have no vertices in common. Such union covers vertex $\mathbf{v}$ if it contains a cycle or a path which covers $\mathbf{v}$.

In the sequel, $\mathcal{S}_{\mathcal{E}}$ represents an edge subset of $\mathcal{E}$ and $\mathcal{V}_{1}$ and $\mathcal{V}_{2}$ two subsets of $\mathcal{V}$. We denote by $\operatorname{card}\left(\mathcal{V}_{1}\right)$ the cardinality of $V_{1}$.

- We say that path $P$ is included in $\mathcal{S}_{\mathcal{E}}$ if all its edges are included in $\mathcal{S}_{\mathcal{E}}$.

- A path $P$ is said a $\mathcal{V}_{1}-\mathcal{V}_{2}$ path if its begin vertex belongs to $\mathcal{V}_{1}$ and its end vertex belongs to $\mathcal{V}_{2}$. If the only vertices of $P$ belonging to $\mathcal{V}_{1} \cup \mathcal{V}_{2}$ are its begin and its end vertices, $P$ is said a direct $V_{1}-V_{2}$ path.

- A set of $l$ disjoint $\mathcal{V}_{1}-\mathcal{V}_{2}$ paths is called a $\mathcal{V}_{1}-\mathcal{V}_{2}$ linking of size $l$. The linkings which consist of a maximal number of disjoint $\mathcal{V}_{1}-\mathcal{V}_{2}$ paths are called maximum $\mathcal{V}_{1}-\mathcal{V}_{2}$ linkings. We define by $\rho\left[\mathcal{V}_{1}, \mathcal{V}_{2}, \mathcal{S}_{\mathcal{E}}\right]$ the size of the maximum $\mathcal{V}_{1}-\mathcal{V}_{2}$ linkings which are included in $\mathcal{S}_{\mathcal{E}}$.

- $\mu\left[\mathcal{V}_{1}, \mathcal{V}_{2}, \mathcal{S}_{\mathcal{E}}\right]$ denotes the minimal number of vertices of $\mathbf{U}_{\mathbf{e}} \cup \mathbf{X} \cup \mathbf{Y}$ covered by a maximum $\mathcal{V}_{1}-\mathcal{V}_{2}$ linking included in $\mathcal{S}_{\mathcal{E}}$.

- $V_{\text {ess }}\left(\mathcal{V}_{1}, \mathcal{V}_{2}, \mathcal{S}_{\mathcal{E}}\right)$ is the vertex subset including the vertices present in all the maximum $\mathcal{V}_{1}-\mathcal{V}_{2}$ linkings included in $\mathcal{S}_{\mathcal{E}}$ and is related to the so-called set of all essential vertices (van der Woude, 2000).

- Vertex subset $\mathbf{S}$ is a separator between sets $\mathcal{V}_{1}$ and $\mathcal{V}_{2}$, if every path from $\mathcal{V}_{1}$ to $\mathcal{V}_{2}$ and included in $\mathcal{S}_{\mathcal{E}}$ contains at least one vertex in $\mathbf{S}$. We call minimum separators between $V_{1}$ and $V_{2}$ any separators having the smallest size. According to Menger's Theorem, the size of all minimum separators between $\mathcal{V}_{1}$ and $\mathcal{V}_{2}$ is equal to $\rho\left[\mathcal{V}_{1}, \mathcal{V}_{2}, \mathcal{S}_{\mathcal{E}}\right]$.

- There exists an uniquely determined minimum separator between $\mathcal{V}_{1}$ and $\mathcal{V}_{2}$ noted $\mathbf{S}\left(\mathcal{V}_{1}, \mathcal{V}_{2}, \mathcal{S}_{\mathcal{E}}\right)$ and called the minimum output separator which is the set of begin vertices of all direct $V_{\text {ess }}\left(\mathcal{V}_{1}, \mathcal{V}_{2}, \mathcal{S}_{\mathcal{E}}\right)-\mathcal{V}_{2}$ paths included in $\mathcal{S}_{\mathcal{E}}$.

\section{Main results}

Using the previous graphic tools, we provide hereafter necessary conditions and next a sufficient one for the generic uniform observability of SBLS $\Sigma_{\Lambda}$.

The two first necessary conditions are based on the fact that the generic uniform observability of SBLS $\Sigma_{\Lambda}$ requires the generic observability of the structured linear system defined by the pair $(A, C)$. The third and last necessary condition, as well as, the sufficient one are less trivial and are related to the strong observability graphic conditions for a structured linear system(Boukhobza et al., 2007).

\subsection{Necessary conditions}

Proposition 3 Consider structured bilinear system $\Sigma_{\Lambda}$ represented by the $\mathcal{G}_{e} . \Sigma_{\Lambda}$ is uniformly observable only if i. each $\mathbf{x}_{\mathbf{i}} \in \mathbf{X}$ is the begin vertex of an $\mathbf{Y}$-topped path included in $\mathcal{E}_{0}$;

ii. there exists a disjoint union of an $\mathbf{Y}$-topped path family and a cycle family included in $\mathcal{E}_{0}$ which covers $\mathbf{X}$;

iii. $\forall \mathbf{V}_{\mathbf{x}} \subseteq \mathbf{X}$,

$\rho\left[\mathbf{V}_{\mathbf{x}} \cup \mathbf{U}, \mathbf{Y}, \mathcal{S}_{\mathcal{E}}\right]>\rho\left[\mathbf{U}, \mathbf{Y}, \mathcal{S}_{\mathcal{E}}\right]$ or $\mu\left[\mathbf{V}_{\mathbf{x}} \cup \mathbf{U}, \mathbf{Y}, \mathcal{S}_{\mathcal{E}}\right]<$ $\mu\left[\mathbf{U}, \mathbf{Y}, \mathcal{S}_{\mathcal{E}}\right]$, where $\mathcal{S}_{\mathcal{E}}=C$-edges $\cup A_{0}$-edges $\cup B_{u}$-edges $\cup$ $\left\{\left(\mathbf{u}_{\mathbf{i}, \mathbf{k}}, \mathbf{x}_{\mathbf{j}}\right) \mid B_{i}(j, k) \neq 0\right.$ and $\left.\mathbf{x}_{\mathbf{k}} \in \mathbf{V}_{\mathbf{x}}\right\}$.

Proof:

i. and ii. $\Sigma_{\Lambda}$ is generically uniformly observable iff all the 
admissible input vector $u$ is universal. Particularly, the input vector constituted by $u_{i}=0, \forall i=1, \ldots m$ must be universal. Therefore, a necessary condition for the generic uniform observability of $\operatorname{SBLS} \Sigma_{\Lambda}$ is that pair $(A, C)$ is observable and so satisfy the well known observability conditions for linear systems recalled in (Dion et al., 2003), which are conditions i and ii of Proposition 3.

iii. Recall first a result on the strong observability of structured linear system (Boukhobza et al., 2007). Consider a structured linear system

$$
\Sigma_{\Lambda, l i n}:\left\{\begin{array}{l}
\dot{x}(t)=A x(t)+B w(t) \\
y(t)=C x(t)
\end{array}\right.
$$

where $w \in R^{q}$ is the input vector. State component $x_{i}$ is strongly observable (Trentelman et al., 2001), only if in the associated digraph of $\Sigma_{\Lambda, \text { lin }}$, we have

$$
\rho\left[\left\{\mathbf{x}_{\mathbf{i}}\right\} \cup \mathbf{W}, \mathbf{Y}, \mathcal{E}\right]>\rho[\mathbf{W}, \mathbf{Y}, \mathcal{E}] \text { or } \mu\left[\left\{\mathbf{x}_{\mathbf{i}}\right\} \cup \mathbf{W}, \mathbf{Y}, \mathcal{E}\right]<\mu[\mathbf{W}, \mathbf{Y}, \mathcal{E}]
$$

This condition can be deduced directly from (Boukhobza et al., 2007) or in a control context from (Commault et al., 1997) where authors treat the disturbance rejection problem. We can also write from condition (4) that, if there exists a vertex subset $\mathbf{V}_{\mathbf{x}} \subseteq \mathbf{X}$, such that

$$
\rho\left[\mathbf{V}_{\mathbf{x}} \cup \mathbf{W}, \mathbf{Y}, \mathcal{E}\right]=\rho[\mathbf{W}, \mathbf{Y}, \mathcal{E}] \text { and } \mu\left[\mathbf{V}_{\mathbf{x}} \cup \mathbf{W}, \mathbf{Y}, \mathcal{E}\right]=\mu[\mathbf{W}, \mathbf{Y}, \mathcal{E}]
$$

then, there exists an input initial condition $w(0)=w^{*}$, such that, $\forall \mathbf{x}_{\mathbf{i}} \in \mathbf{V}_{\mathbf{x}}, y(t)=0$ for $t \geq 0$ does not imply that $x_{i}(0)=0$, or in other words that all state components associated to $\mathbf{x}_{\mathbf{i}} \in \mathbf{V}_{\mathbf{x}}$ are not strongly observable. Thus, if there exists a vertex subset $\mathbf{V}_{\mathbf{x}} \subseteq \mathbf{X}$ such that condition (5) is satisfied, then there exist $w(0)=w^{*}$ and initial conditions $x_{i}(0) \neq 0, \forall \mathbf{x}_{\mathbf{i}} \in \mathbf{V}_{\mathbf{x}}$, such that $y(t)=0, \forall t \geq 0$.

Let us come back to structured bilinear system $\Sigma_{\Lambda}$ and assume that condition iii. is not satisfied i.e. $\exists \mathbf{V}_{\mathbf{x}} \subseteq \mathbf{X}$, $\rho\left[\mathbf{V}_{\mathbf{x}} \cup \mathbf{U}, \mathbf{Y}, \mathcal{S}_{\mathcal{E}}\right]=\rho\left[\mathbf{U}, \mathbf{Y}, \mathcal{S}_{\mathcal{E}}\right]$ and $\mu\left[\mathbf{V}_{\mathbf{x}} \cup \mathbf{U}, \mathbf{Y}, \mathcal{S}_{\mathcal{E}}\right]=$ $\mu\left[\mathbf{U}, \mathbf{Y}, \mathcal{S}_{\mathcal{E}}\right]$, where $\mathcal{S}_{\mathcal{E}}=C$-edges $\cup A_{0}$-edges $\cup B_{u}$-edges $\cup$ $\left\{\left(\mathbf{u}_{\mathbf{i}, \mathbf{k}}, \mathbf{x}_{\mathbf{j}}\right) \mid B_{i}(j, k) \neq 0\right.$ and $\left.\mathbf{x}_{\mathbf{k}} \in \mathbf{V}_{\mathbf{x}}\right\}$.

Let us denote by $\overline{\mathbf{U}} \subseteq \mathbf{U}$ a vertex subset such that $\rho\left[\mathbf{V}_{\mathbf{x}} \cup \overline{\mathbf{U}}, \mathbf{Y}, \mathcal{S}_{\mathcal{E}}\right]=\rho\left[\overline{\mathbf{U}}, \mathbf{Y}, \mathcal{S}_{\mathcal{E}}\right]=\rho\left[\mathbf{U}, \mathbf{Y}, \mathcal{S}_{\mathcal{E}}\right]=\operatorname{card}(\overline{\mathbf{U}})$ and $\mu\left[\mathbf{V}_{\mathbf{x}} \cup \overline{\mathbf{U}}, \mathbf{Y}, \mathcal{S}_{\mathcal{E}}\right]=\mu\left[\overline{\mathbf{U}}, \mathbf{Y}, \mathcal{S}_{\mathcal{E}}\right]=\mu\left[\mathbf{U}, \mathbf{Y}, \mathcal{S}_{\mathcal{E}}\right]$. Subset $\overline{\mathbf{U}}$ always exists and corresponds to the set of begin vertices of the maximal linking of minimal size included in $\mathcal{S}_{\mathcal{E}}$ between $\mathbf{U}$ and $\mathbf{Y}$. Note that $\overline{\mathbf{U}}$ is not necessarily unique.

Naturally, since all the inputs are independent, we can assign any independent values to $u_{i}(0), \mathbf{u}_{\mathbf{i}} \in \overline{\mathbf{U}}$. Let us denote $w_{i} \stackrel{\text { def }}{=} u_{i} x_{k}$, where $\mathbf{u}_{\mathbf{i}} \in \overline{\mathbf{U}}$ and $\mathbf{x}_{\mathbf{k}} \in \mathbf{V}_{\mathbf{x}}$ are chosen such that a maximal linking $\overline{\mathbf{U}}-\mathbf{Y}$ of minimal size pass through $\mathbf{u}_{\mathbf{i}, \mathbf{k}}$. According to the previous discussion on linear systems, there exist values $w_{i}(0)=w_{i}^{*}$ and initial conditions $x_{j}(0) \neq 0, \forall \mathbf{x}_{\mathbf{j}} \in \mathbf{V}_{\mathbf{x}}$, such that $y(t)=0, \forall t \geq 0$. By definition of $w_{i}$, when $x_{j}(0) \neq 0, \forall \mathbf{x}_{\mathbf{j}} \in \mathbf{V}_{\mathbf{x}}$, we can assign any desired value $w_{i}^{d}$ to the $\operatorname{card}(\overline{\mathbf{U}})$ elements $w_{i}(0)$ using as control input value $u_{i}(0)=\frac{w_{i}^{d}}{x_{k}(0)}$ because $\mathbf{x}_{\mathbf{k}} \in \mathbf{V}_{\mathbf{x}}$. In particular, we can assign to $w_{i}(0)$ the value $w_{i}^{*}$ which makes all the state components associated to $\mathbf{x}_{\mathbf{j}} \in \mathbf{V}_{\mathbf{x}}$ indistinguishable from 0 . Hence, if condition iii is not satisfied, then there exist $x_{j}(0) \neq 0, \forall \mathbf{x}_{\mathbf{j}} \in \mathbf{V}_{\mathbf{x}}$ and $u_{i}(0)=\frac{w_{i}^{*}}{x_{k}(0)}$ (knowing that $x_{k}(0) \neq 0$ as $\left.\mathbf{x}_{\mathbf{k}} \in \mathbf{V}_{\mathbf{x}}\right)$, such that $y(t)=0, \forall t \geq 0$. Therefore, there exist generically control values such that the initial states are not distinguishable from 0 and so condition iii is necessary to the generic uniform observability of system $\Sigma_{\Lambda}$.

Consider Example 2 and let us check the conditions of Proposition 3 above. We have:

- Condition i: all state vertices are the end vertices of an Y-topped path,

- Condition ii: there exists an Y-topped path family included in $\mathcal{E}_{0}$ which covers $\mathbf{X}$ (in the present case, we do not need a cycle family) $: \mathbf{x}_{5} \rightarrow \mathbf{x}_{3} \rightarrow \mathbf{x}_{1} \rightarrow \mathbf{y}_{1}$ and $\mathbf{x}_{4} \rightarrow \mathbf{x}_{2} \rightarrow \mathbf{y}_{2}$.

- condition iii: On the one hand, $\forall \mathbf{V}_{\mathbf{x}} \subseteq \mathbf{X}$ such that $\left\{\mathbf{x}_{\mathbf{2}}, \mathbf{x}_{\mathbf{5}}\right\} \not \subset \mathbf{V}_{\mathbf{x}}$, using notations of Proposition 3 for $\mathcal{S}_{\mathcal{E}}$, we have $2=\rho\left[\mathbf{V}_{\mathbf{X}} \cup \mathbf{U}, \mathbf{Y}, \mathcal{S}_{\mathcal{E}}\right]>\rho\left[\mathbf{U}, \mathbf{Y}, \mathcal{S}_{\mathcal{E}}\right]=1$.

On the other hand, $\forall \mathbf{V}_{\mathbf{x}} \subseteq \mathbf{X}$ such that $\left\{\mathbf{x}_{\mathbf{2}}, \mathbf{x}_{\mathbf{5}}\right\} \subseteq \mathbf{V}_{\mathbf{x}}$, we have $\rho\left[\mathbf{V}_{\mathbf{x}} \cup \mathbf{U}, \mathbf{Y}, \mathcal{S}_{\mathcal{E}}\right]=\rho\left[\mathbf{U}, \mathbf{Y}, \mathcal{S}_{\mathcal{E}}\right]=2$ and $\mu\left[\mathbf{V}_{\mathbf{x}} \cup\right.$ $\left.\mathbf{U}, \mathbf{Y}, \mathcal{S}_{\mathcal{E}}\right]<\mu\left[\mathbf{U}, \mathbf{Y}, \mathcal{S}_{\mathcal{E}}\right]=6$. Indeed, if $\mathbf{x}_{\mathbf{1}}$ belongs to $\mathbf{V}_{\mathbf{x}}$, $\mu\left[\mathbf{V}_{\mathbf{x}} \cup \mathbf{U}, \mathbf{Y}, \mathcal{S}_{\mathcal{E}}\right]=4$, else $\mu\left[\mathbf{V}_{\mathbf{x}} \cup \mathbf{U}, \mathbf{Y}, \mathcal{S}_{\mathcal{E}}\right]=5$. Hence, condition iii is also satisfied.

The uniform observability implies a constraint which must be satisfied for any input value. So, it is quite logical that a necessary and sufficient condition must not depend on the input values. In this context, it can be questionable that condition iii of Proposition 3 depends on the input vertices. On the one hand, in our digraph the vertices do not represent only the input values. In fact, the notion of input values in not explicite in the digraph representation while the input vertices reflect essentially the system's structure. Indeed, condition iii of Proposition 3 is based on the comparison of the "distance" between the input vertices and the output vertices to the distance between the state and the output vertices. This comparison is purely structural and does not traduce any dependence on the input values. Yet, the uniform observability is a property which depends strongly on the system's structure and on the matrices $A$ and $B_{i}$, $i=1, \ldots, m$. Moreover, there exists a necessary condition which does not depend on the input vertices. This is the case of conditions $\mathbf{i}$ and $\mathbf{i i}$ which do not depend on the input vertices.

\subsection{Sufficient condition}

Before giving the sufficient condition for the generic uniform observability, let us first specify a particular subdivision of structured system $\Sigma_{\Lambda}$ :

Definition 4 Consider an edge subset $\mathcal{S}_{\mathcal{E}} \subseteq \mathcal{E}$, for each vertex subsets $\mathbf{V}$ such that $\mathbf{Y} \subseteq \mathbf{V} \subseteq \mathbf{X} \cup \mathbf{Y}$, we define the following vertex subsets:

$\overline{\mathbf{X}}(\mathbf{V})=\mathbf{X} \backslash(\mathbf{V} \cap \mathbf{X}), \overline{\mathbf{U}}\left(\mathbf{V}, \mathcal{S}_{\mathcal{E}}\right) \subseteq \mathbf{U}_{\mathbf{e}}$ such that $\operatorname{card}\left(\overline{\mathbf{U}}\left(\mathbf{V}, \mathcal{S}_{\mathcal{E}}\right)\right)=$ $\rho\left[\mathbf{U}_{\mathbf{e}}, \mathbf{V}, \mathcal{S}_{\mathcal{E}}\right]=\rho\left[\overline{\mathbf{U}}\left(\mathbf{V}, \mathcal{S}_{\mathcal{E}}\right), \mathbf{V}, \mathcal{S}_{\mathcal{E}}\right]$ and $\mu\left[\overline{\mathbf{U}}\left(\mathbf{V}, \mathcal{S}_{\mathcal{E}}\right), \mathbf{V}, \mathcal{S}_{\mathcal{E}}\right]=$ $\mu\left[\mathbf{U}_{\mathbf{e}}, \mathbf{V}, \mathcal{S}_{\mathcal{E}}\right]$. Note that $\overline{\mathbf{U}}\left(\mathbf{V}, \mathcal{S}_{\mathcal{E}}\right)$ always exists but is not necessarily unique.

$$
\begin{aligned}
& \mathbf{X}_{\mathbf{1}}\left(\mathbf{V}, \mathcal{S}_{\mathcal{E}}\right) \stackrel{\text { def }}{=}\left\{\mathbf{x}_{\mathbf{i}} \in \overline{\mathbf{X}}(\mathbf{V}) \mid \rho\left[\mathbf{U}_{\mathbf{e}} \cup\left\{\mathbf{x}_{\mathbf{i}}\right\}, \mathbf{V}, \mathcal{S}_{\mathcal{E}}\right]>\rho\left[\mathbf{U}_{\mathbf{e}}, \mathbf{V}, \mathcal{S}_{\mathcal{E}}\right]\right\}, \\
& \boldsymbol{\Upsilon}_{\mathbf{0}}\left(\mathbf{V}, \mathcal{S}_{\mathcal{E}}\right) \stackrel{\text { def }}{=} \mathbf{V} \cap V_{\text {ess }}\left(\mathbf{U}_{\mathbf{e}}, \mathbf{V}, \mathcal{S}_{\mathcal{E}}\right),
\end{aligned}
$$


$\boldsymbol{\Upsilon}_{\mathbf{1}}\left(\mathbf{V}, \mathcal{S}_{\mathcal{E}}\right) \stackrel{\text { def }}{=} \mathbf{V} \backslash \boldsymbol{\Upsilon}_{\mathbf{0}}\left(\mathbf{V}, \mathcal{S}_{\mathcal{E}}\right)$

$\mathbf{U}_{\mathbf{0}}\left(\mathbf{V}, \mathcal{S}_{\mathcal{E}}\right) \stackrel{\text { def }}{=}\left\{\mathbf{u}_{\mathbf{i}, \mathbf{j}} \in \overline{\mathbf{U}}\left(\mathbf{V}, \mathcal{S}_{\mathcal{E}}\right) \mid \mu\left[\left\{\mathbf{u}_{\mathbf{i}}\right\}, \mathbf{X}_{\mathbf{1}}\left(\mathbf{V}, \mathcal{S}_{\mathcal{E}}\right), \mathcal{S}_{\mathcal{E}}\right]>2\right\}$,

$\mathbf{U}_{\mathbf{1}}\left(\mathbf{V}, \mathcal{S}_{\mathcal{E}}\right) \stackrel{\text { def }}{=} \overline{\mathbf{U}}\left(\mathbf{V}, \mathcal{S}_{\mathcal{E}}\right) \backslash \mathbf{U}_{\mathbf{0}}\left(\mathbf{V}, \mathcal{S}_{\mathcal{E}}\right)$,

$\mathbf{S}^{*}\left(\mathbf{V}, \mathcal{S}_{\mathcal{E}}\right)=\mathbf{S}^{\mathbf{o}}\left(\mathbf{U}_{\mathbf{0}}\left(\mathbf{V}, \mathcal{S}_{\mathcal{E}}\right), \mathbf{V}, \mathcal{S}_{\mathcal{E}}\right), \mathbf{X}_{\mathbf{s}}\left(\mathbf{V}, \mathcal{S}_{\mathcal{E}}\right) \stackrel{\text { def }}{=} \mathbf{S}^{*}\left(\mathbf{V}, \mathcal{S}_{\mathcal{E}}\right) \cap$ $\overline{\mathbf{X}}(\mathbf{V})$.

We define $\beta_{1}\left(\mathbf{V}, \mathcal{S}_{\mathcal{E}}\right)$ as the maximal number of vertices included in $\mathbf{X}_{\mathbf{1}}\left(\mathbf{V}, \mathcal{S}_{\mathcal{E}}\right) \cup \mathbf{X}_{\mathbf{s}}\left(\mathbf{V}, \mathcal{S}_{\mathcal{E}}\right) \cup \mathbf{U}_{\mathbf{1}}\left(\mathbf{V}, \mathcal{S}_{\mathcal{E}}\right)$ covered by an union of

- a linking of size $\rho\left[\mathbf{X}_{\mathbf{s}}\left(\mathbf{V}, \mathcal{S}_{\mathcal{E}}\right) \cup \mathbf{U}_{\mathbf{1}}\left(\mathbf{V}, \mathcal{S}_{\mathcal{E}}\right), \Upsilon_{\mathbf{1}}\left(\mathbf{V}, \mathcal{S}_{\mathcal{E}}\right), \mathcal{S}_{\mathcal{E}}\right]$

from $\mathbf{X}_{\mathbf{s}}\left(\mathbf{V}, \mathcal{S}_{\mathcal{E}}\right) \cup \mathbf{U}_{\mathbf{1}}\left(\mathbf{V}, \mathcal{S}_{\mathcal{E}}\right)$ to $\boldsymbol{\Upsilon}_{\mathbf{1}}\left(\mathbf{V}, \mathcal{S}_{\mathcal{E}}\right)$,

- an $\boldsymbol{\Upsilon}_{\mathbf{1}}\left(\mathbf{V}, \mathcal{S}_{\mathcal{E}}\right)$-topped path family and

- a cycle family covering only elements of $\mathbf{X}_{\mathbf{1}}\left(\mathbf{V}, \mathcal{S}_{\mathcal{E}}\right)$.

Finally,

$\beta\left(\mathbf{V}, \mathcal{S}_{\mathcal{E}}\right) \stackrel{\text { def }}{=} \beta_{1}\left(\mathbf{V}, \mathcal{S}_{\mathcal{E}}\right)+\mu\left[\mathbf{U}_{\mathbf{0}}\left(\mathbf{V}, \mathcal{S}_{\mathcal{E}}\right), \mathbf{S}^{*}\left(\mathbf{V}, \mathcal{S}_{\mathcal{E}}\right), \mathcal{S}_{\mathcal{E}}\right]-$ $\rho\left[\mathbf{U}_{\mathbf{0}}\left(\mathbf{V}, \mathcal{S}_{\mathcal{E}}\right), \mathbf{S}^{*}\left(\mathbf{V}, \mathcal{S}_{\mathcal{E}}\right), \mathcal{S}_{\mathcal{E}}\right]$.

Using the previous definition, we state the following lemma:

Lemma 5 Consider structured system $\Sigma_{\Lambda}$ represented by digraph $\mathcal{G}_{e} . \forall i=1, \ldots, n$, if

$\beta\left(\mathbf{Y} \cup\left\{\mathbf{x}_{\mathbf{i}}\right\}, \mathcal{E}_{1}\right)=\beta\left(\mathbf{Y}, \mathcal{E}_{1}\right)$, where $\mathcal{E}_{1}=\bigcup_{i=0}^{m} A_{i}$-edges $\cup$

$C$-edges $\cup B$-edges, then state component $x_{i}$ is generically uniformly observable.

Proof: Consider structured linear system $\Sigma_{\Lambda, l i n}$. It was proved in (Boukhobza et al., 2007) on the basis of the results of (van der Woude, 2000) that:

St1. The state component $x_{i}$ is generically strongly observable iff $\beta\left(\mathbf{Y} \cup\left\{\mathbf{x}_{\mathbf{i}}\right\}, \mathcal{E}\right)=\beta(\mathbf{Y}, \mathcal{E})$. This implies that if $\beta\left(\mathbf{Y} \cup\left\{\mathbf{x}_{\mathbf{i}}\right\}, \mathcal{E}\right)=\beta(\mathbf{Y}, \mathcal{E})$ then for all input function $w(0)$, $y(t)=0, \forall t \geq 0$ imply that $x_{i}(0)=0$.

Applying this for the structured bilinear system $\Sigma_{\Lambda}$, and considering each of the terms $u_{l} x_{j}$ as an independent input $w_{k}$, we have that $\beta\left(\mathbf{Y} \cup\left\{\mathbf{x}_{\mathbf{i}}\right\}, \mathcal{E}_{1}\right)=\beta\left(\mathbf{Y}, \mathcal{E}_{1}\right)$ ensures, for all values of $w_{k}(0)$ and so of $u_{j}(0) x_{k}(0)$, that $y(t)=0, \forall t \geq 0$ implies that $x_{i}(0)=0$. This means that there does not exist an input vector $u(0)$ for which two initial states components $x_{i}(0)$ and $x_{i}^{\prime}(0)$ are indistinguishable. Hence, component $x_{i}$ is generically uniformly observable.

Now, we present an algorithm which allows to precise iteratively the set of uniformly observable state components:

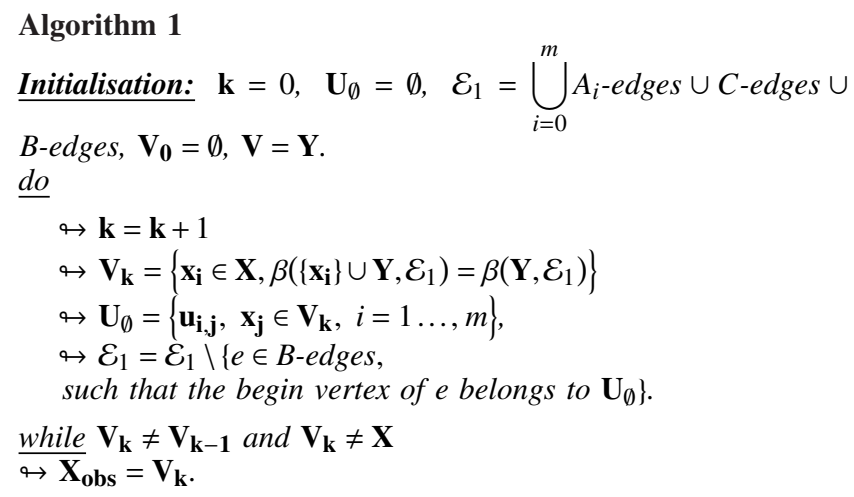

Corollary 6 Consider SBLS $\Sigma_{\Lambda}$ represented by digraph $\mathcal{G}_{e}$ and vertex subset $\mathbf{X}_{\mathbf{o b s}}$ computed by Algorithm 1. If $\mathbf{X}_{\mathbf{o b s}}=\mathbf{X}$ then $\Sigma_{\Lambda}$ is generically uniformly observable.
Proof: We have proved in Lemma 5 that, after the first loop of Algorithm 1, the vertices included in $\mathbf{V}_{\mathbf{1}}$ are associated to generically uniformly observable components. Thus, all the vertices $\left\{\mathbf{u}_{\mathbf{i}, \mathbf{j}}, \mathbf{x}_{\mathbf{j}} \in \mathbf{V}_{\mathbf{1}}, i=1 \ldots, m\right\}$ correspond to products $u_{i} x_{j}$ where $x_{j}$ is uniformly observable and so these products can be expressed only function of known variables $u$ and $y$ and their derivatives. Thus, they have no effect on the uniform observability of the system. In this case we can remove the corresponding vertices $u_{i, j}$ from the considered digraph which is equivalent to take only into account a subset of $\mathcal{E}_{1}$ which is $\mathcal{E}_{1} \backslash\left(\left\{\left(\mathbf{u}_{\mathbf{i}, \mathbf{j}}, \mathbf{x}_{\mathbf{k}}\right), \mathbf{x}_{\mathbf{j}} \in \mathbf{V}_{\mathbf{1}}, i=1 \ldots, m\right\}\right)$, which corresponds to $\mathcal{E}_{1}=\mathcal{E}_{1} \backslash\left\{e \in B\right.$-edges, such that the begin vertex of $\left.e \in \mathbf{U}_{\emptyset}\right\}$. We can repeat the computation of the state vertex subset $\left\{\mathbf{x}_{\mathbf{i}} \in \mathbf{X}, \beta\left(\left\{\mathbf{x}_{\mathbf{i}}\right\} \cup \mathbf{Y}, \mathcal{E}_{1}\right)=\beta\left(\mathbf{Y}, \mathcal{E}_{1}\right)\right\}$ denoted $\mathbf{V}_{\mathbf{2}}$. We can then apply as previously Lemma 5 , to ensure that the elements of subset $\mathbf{V}_{2}$ are associated to generically uniformly observable state components. Thus, iteratively, all the state vertices including in $\mathbf{V}_{\mathbf{k}}$ are associated to uniformly observable state components.

We repeat the computation of subsets $\mathbf{V}_{\mathbf{k}}$ until no new state component is added to $\mathbf{V}_{\mathbf{k}}$ i.e. $\mathbf{V}_{\mathbf{k}}=\mathbf{V}_{\mathbf{k}-\mathbf{1}}$ or until $\mathbf{V}_{\mathbf{k}}=\mathbf{X}$ which implies that all the state components are generically uniformly observable.

Note that by construction, $\mathbf{V}_{\mathbf{k}-\mathbf{1}} \subseteq \mathbf{V}_{\mathbf{k}} \subseteq \mathbf{X}$. This ensures obviously that Algorithm 1 converges at most in $n$ steps. $\triangle$ Roughly speaking, the presented sufficient conditions ensure that the system can be transformed into some triangular form (Boukhobza et al., 2006), which ensures the uniform observability and which is used in the design of many nonlinear observers. Moreover, the proposed sufficient condition in Corollary 6 are equivalent to the ones presented in (Boukhobza et al., 2006) but the latter are very complicated and have an exponential complexity order. Let us now illustrate Algorithm 1 on Example 2:

Initialisation: $\mathbf{k}=0, \mathbf{U}_{\emptyset}=\emptyset, \mathcal{E}_{1}=C$-edges $\cup A$-edges $\cup B$-edges,

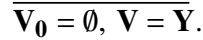

$\leftrightarrow \mathbf{k}=1$,

$\leftrightarrow \beta\left(\mathbf{Y}, \mathcal{E}_{1}\right)=\beta\left(\mathbf{Y} \cup\left\{\mathbf{x}_{\mathbf{1}}\right\}, \mathcal{E}_{1}\right)=\beta\left(\mathbf{Y} \cup\left\{\mathbf{x}_{\mathbf{2}}\right\}, \mathcal{E}_{1}\right)=4$,

$\beta\left(\mathbf{Y} \cup\left\{\mathbf{x}_{\mathbf{3}}\right\}, \mathcal{E}_{1}\right)=6, \beta\left(\mathbf{Y} \cup\left\{\mathbf{x}_{\mathbf{4}}\right\}, \mathcal{E}_{1}\right)=\beta\left(\mathbf{Y} \cup\left\{\mathbf{x}_{\mathbf{5}}\right\}, \mathcal{E}_{1}\right)=5$,

so $\mathbf{V}_{\mathbf{1}}=\left\{\mathbf{x}_{\mathbf{1}}, \mathbf{x}_{\mathbf{2}}\right\}$ and $\mathbf{U}_{\emptyset}=\left\{\mathbf{u}_{\mathbf{2}, 2}\right\}$,

$\leftrightarrow \mathcal{E}_{1}=C$-edges $\cup A$-edges $\cup B$-edges $\backslash\left\{\left(\mathbf{u}_{2,2}, \mathbf{x}_{1}\right)\right\}$,

as $\mathbf{V}_{\mathbf{1}} \neq \mathbf{V}_{\mathbf{0}}$ and $\mathbf{V}_{\mathbf{1}} \neq \mathbf{X}$ we continue

$\rightarrow \mathbf{k}=2$,

$\leftrightarrow \beta\left(\mathbf{Y}, \mathcal{E}_{1}\right)=\beta\left(\mathbf{Y} \cup\left\{\mathbf{x}_{\mathbf{1}}\right\}, \mathcal{E}_{1}\right)=\beta\left(\mathbf{Y} \cup\left\{\mathbf{x}_{\mathbf{2}}\right\}, \mathcal{E}_{1}\right)=\beta\left(\mathbf{Y} \cup\left\{\mathbf{x}_{\mathbf{3}}\right\}, \mathcal{E}_{1}\right)=$

$5, \beta\left(\mathbf{Y} \cup\left\{\mathbf{x}_{\mathbf{4}}\right\}, \mathcal{E}_{1}\right)=6, \beta\left(\mathbf{Y} \cup\left\{\mathbf{x}_{\mathbf{5}}\right\}, \mathcal{E}_{1}\right)=6$, so $\mathbf{V}_{\mathbf{2}}=\left\{\mathbf{x}_{\mathbf{1}}, \mathbf{x}_{\mathbf{2}}, \mathbf{x}_{\mathbf{3}}\right\}$,

$\leftrightarrow \mathbf{U}_{\emptyset}=\left\{\mathbf{u}_{2,2}, \mathbf{u}_{1,3}\right\}$,

$\leftrightarrow \mathcal{E}_{1}=C$-edges $\cup A$-edges $\cup B$-edges $\backslash\left\{\left(\mathbf{u}_{\mathbf{2}, \mathbf{2}}, \mathbf{x}_{\mathbf{1}}\right),\left(\mathbf{u}_{\mathbf{1}, \mathbf{3}}, \mathbf{x}_{\mathbf{3}}\right)\right\}$,

as $\mathbf{V}_{\mathbf{2}} \neq \mathbf{V}_{\mathbf{1}}$ and $\mathbf{V}_{\mathbf{2}} \neq \mathbf{X}$ we continue

$\leftrightarrow \mathbf{k}=3$,

$\leftrightarrow \beta\left(\mathbf{Y}, \mathcal{E}_{1}\right)=\beta\left(\mathbf{Y} \cup\left\{\mathbf{x}_{\mathbf{1}}\right\}, \mathcal{E}_{1}\right)=\beta\left(\mathbf{Y} \cup\left\{\mathbf{x}_{\mathbf{2}}\right\}, \mathcal{E}_{1}\right)=\beta\left(\mathbf{Y} \cup\left\{\mathbf{x}_{\mathbf{3}}\right\}, \mathcal{E}_{1}\right)=$

$\beta\left(\mathbf{Y} \cup\left\{\mathbf{x}_{\mathbf{5}}\right\}, \mathcal{E}_{1}\right)=5 \beta\left(\mathbf{Y} \cup\left\{\mathbf{x}_{\mathbf{4}}\right\}, \mathcal{E}_{1}\right)=6$, so $\mathbf{V}_{\mathbf{3}}=\left\{\mathbf{x}_{\mathbf{1}}, \mathbf{x}_{\mathbf{2}}, \mathbf{x}_{\mathbf{3}}, \mathbf{x}_{\mathbf{5}}\right\}$,

$\leftrightarrow \mathbf{U}_{\emptyset}=\left\{\mathbf{u}_{2,2}, \mathbf{u}_{1,3}, \mathbf{u}_{1,5}\right\}$,

$\leftrightarrow \mathcal{E}_{1}=C$-edges $\cup A$-edges $\cup B$-edges $\backslash\left\{\left(\mathbf{u}_{\mathbf{2}, \mathbf{2}}, \mathbf{x}_{\mathbf{1}}\right),\left(\mathbf{u}_{\mathbf{1}, \mathbf{3}}, \mathbf{x}_{\mathbf{3}}\right)\right.$,

$\left.\left(\mathbf{u}_{1,5}, \mathbf{x}_{2}\right)\right\}$,

as $\mathbf{V}_{\mathbf{3}} \neq \mathbf{V}_{\mathbf{2}}$ and $\mathbf{V}_{\mathbf{3}} \neq \mathbf{X}$ we continue

$\leftrightarrow \mathbf{k}=4$,

$\leftrightarrow \forall \mathbf{x}_{\mathbf{i}} \in \mathbf{X}, \beta\left(\mathbf{Y}, \mathcal{E}_{1}\right)=\beta\left(\mathbf{Y} \cup\left\{\mathbf{x}_{\mathbf{i}}\right\}, \mathcal{E}_{1}\right)=5$ and so $\mathbf{V}_{\mathbf{4}}=\mathbf{X}$ 
$\leftrightarrow \mathbf{U}_{\emptyset}=\mathbf{U}_{\mathbf{e}}$,

$\leftrightarrow \mathcal{E}_{1}=C$-edges $\cup A$-edges,

as $\mathbf{V}_{\mathbf{4}}=\mathbf{X}$ we stop. $\mathbf{X}_{\mathbf{o b s}}=\mathbf{X}$ implies that the system is generically uniformly observable.

The following example illustrates some of the improvements of the present paper w.r.t. the results of (Boukhobza et al., 2006).

Example 7 Consider the SBLS represented by the digraph depicted in Figure 2 The conditions $\mathbf{i}$ and $\mathbf{i i}$ of Proposition 3

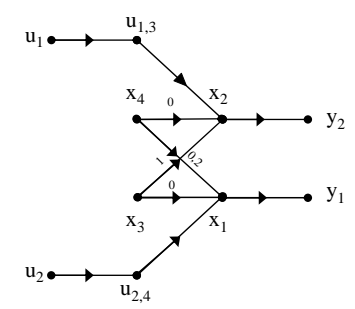

Figure 2. Digraph associated to the system of Example 7

are satisfied and so also the necessary conditions enounced in (Boukhobza et al., 2006). Indeed, every state component is the begin of an $\mathbf{Y}$-topped path and there exist a disjoint union of an $\mathbf{Y}$-topped path family and a cycle family included in $\mathcal{E}_{0}$ which covers $\mathbf{X}: \mathbf{x}_{\mathbf{3}} \rightarrow \mathbf{x}_{\mathbf{1}} \rightarrow \mathbf{y}_{1}$ and $\mathbf{x}_{\mathbf{4}} \rightarrow \mathbf{x}_{\mathbf{2}} \rightarrow \mathbf{y}_{\mathbf{2}}$. Nevertheless, condition iii is not satisfied because for $\mathbf{V}_{\mathbf{x}}=$ $\left\{\mathbf{x}_{\mathbf{3}}, \mathbf{x}_{\mathbf{4}}\right\}$, we have $\rho\left[\mathbf{V}_{\mathbf{x}} \cup \mathbf{U}, \mathbf{Y}, \mathcal{S}_{\mathcal{E}}\right]=\rho\left[\mathbf{U}, \mathbf{Y}, \mathcal{S}_{\mathcal{E}}\right]=2$ and $\mu\left[\mathbf{V}_{\mathbf{x}} \cup \mathbf{U}, \mathbf{Y}, \mathcal{S}_{\mathcal{E}}\right]=\mu\left[\mathbf{U}, \mathbf{Y}, \mathcal{S}_{\mathcal{E}}\right]=6$, where $\mathcal{S}_{\mathcal{E}}=C$-edges $\cup$ $A_{0}$-edges $\cup B_{u^{-}}$-edges $\cup\left\{\left(\mathbf{u}_{\mathbf{i}, \mathbf{k}}, \mathbf{x}_{\mathbf{j}}\right) \mid A_{i}(j, k) \neq 0\right.$ et $\left.\mathbf{x}_{\mathbf{k}} \in \mathbf{V}_{\mathbf{x}}\right\}$ i.e. $\mathcal{S}_{\mathcal{E}}=E \backslash\left(A_{1}\right.$-edges $\cup A_{2}$-edges $)$. Thus, while Proposition 4 allows us to affirm that this system is not generically uniformly observable, the results enounced in (Boukhobza et al., 2006) does not enable us to give any conclusion on the uniform observability of system.

Another improvement of the conditions enounced in the present paper w.r.t. the ones provided in (Boukhobza et al., 2006) is the complexity order of the sufficiency condition. Indeed, in (Boukhobza et al., 2006) the proposed algorithm which allows to compute the so-called permitted vertices has an exponential complexity order. Even if the condition of Corollary 6 is equivalent, in the mathematical sens, to the sufficient condition of (Boukhobza et al., 2006), its implementation necessitates an algorithm with a polynomial complexity order as it is discussed in the conclusion.

\section{Conclusion}

In this paper, we propose an analysis tool to study the generic uniform observability of structured bilinear systems. Using a graphic representation dedicated to this class of nonlinear systems, some necessary and a sufficient conditions are provided and expressed in graphic terms. These conditions are far to be trivial and are obviously necessary and sufficient in the single output case. Furthermore they need few information about the system and are very easy to check by means of well-known combinatorial techniques and simply by hand for small systems. That makes our approach particularly suited for large-scale and sparse systems as it is free from numerical difficulties. Indeed, from a computational point of view, the necessary conditions enounced in Proposition 3 use algorithms which complexity order are, respectively,
$O\left(n \times \log _{2} n\right)$ for condition $\mathbf{i}$ and $O\left(n^{3 / 2}\right)$ by using the Bipmatch method (Micali and Vazirani, 1980) for condition ii. Condition iii requires first a reduction of the digraph in function of the minimal length paths between $\mathbf{U}_{\mathbf{e}}$ and $\mathbf{Y}$. This allows to obtain an algorithm which complexity order is equal to $O\left(M \times N^{2}\right)$ where $M=n^{2}+2 n^{2} m+n m^{2}+n p$ is the number of edges and $N=n(1+m)+p$ is the number of vertices in the digraph. Furthermore, the algorithm used to check the sufficient condition is constituted by at most $n(n+1) / 2$ computations of function $\beta$. To compute such function, we use an algorithm which complexity order equals $O\left(N^{3} \times M^{0.5}\right)$ (Boukhobza et al., 2007; Martinez-Martinez et al., 2006) using the primal-dual algorithm (Hovelaque et al., 1996).

\section{References}

Bornard, G. \& Hammouri, H. (2002). A graph approach to uniform observability of linear multi output systems. Proceedings of the $41^{\text {st }}$ IEEE Conference on Decision and Control, 701-706, Las Vegas, U.S.A.

Boukhobza, T., Hamelin, F. \& Martinez-Martinez, S. (2007). State and input observability for structured linear systems: A graph-theoretic approach. Automatica, 43(7), 1204-1210.

Boukhobza, T., Hamelin, F. \& Sauter, D. (2006). Uniform observability analysis for structured bilinear systems: A graph-theoretic approach. European Journal of Control, 12(5), 505-518.

Commault, C., Dion, J-M., \& Hovelaque, V. (1997). A geometric approach for structured systems: Application to disturbance decoupling. Automatica, 33(3), 403-409.

Dion, J-M., Commault, C. \& van der Woude, J. (2003). Generic properties and control of linear structured systems: A survey. Automatica, 39(7), $1125-1144$.

Gauthier, J-P. \& Bornard, G. (1981). Observability for any $u(t)$ of a class of nonlinear systems. IEEE Transactions on Automatic Control, 26(4), 922-926.

Gauthier, J-P. \& Kupka, I. A. K. (1994). Observability and observers for nonlinear systems. SIAM Journal of Control and Optimization, 32(4), 975-994.

Grasselli, O. M. \& Isidori, A. (1977). Deterministic state reconstruction and reachability of bilinear processes. Proceedings of IEEE Joint Automatic Control Conference, 1423-1427, San Francisco, U.S.A.

Hermann, R. \& Krener, A. J. Nonlinear controllability and observability. (1977). IEEE Transactions on Automatic Control, 22(6), 728-740.

Hovelaque, V., Commault, C. \& Dion, J-M. (1996). Analysis of linear structured systems using a primal-dual algorithm. System $\mathcal{E}$ Control Letters, 27(2), 73-85.

Kou, S. R., Elliot, D. L. \& Tarn, T. J. (1996). Observability of nonlinear systems. Information and Control, 22, 89-99.

Lévine, J. (1997) A graph-theoretic approach to input output decoupling and linearization. Nonlinear Systems, Chapter 3, 77-91. Edited by Fossard A. J. \& Normand-Cyrot, D. Chapman \& Hall, London, U.K.

Martinez-Martinez, S. Mader, T., Boukhobza, T. \& Hamelin. F. (2006). LISA: A linear structured system analysis program. Proceedings of the German-French Institute for Automation and Robotics Annual Meeting, Nancy, France.

Micali, S. \& Vazirani, V. V. (1980). An $O\left(\left|V^{1 / 2} E\right|\right)$ algorithm for finding maximum matching in general graphs. In Proceedings of the $21^{\text {st }}$ Annual Symposium on the Foundations of Computer Science, 17-27.

Mohler, R. R. (1991). Nonlinear Systems: Application to Bilinear Systems. Prentice-Hall, Englewood Cliffs, U.S.A.

Svaricek, F. (1993). A graph theoretic approach for the investigation of the observability of bilinear systems. Proceedings of the $12^{\text {th }}$ IFAC World Congress, 351-354, Sydney, Australia.

Trentelman, H. L., Stoorvogel, A. A. \& Hautus, M. (2001). Control Theory for Linear Systems. Springer, London, U.K.

van der Woude, J. W. (2000). The generic number of invariant zeros of a structured linear system. SIAM Journal of Control and Optimization, $38(1), 1-21$.

Williamson, D. (1977). Observation of bilinear systems with application to biological control. Automatica, 13(3), 243-254. 